\title{
Recursive procedure in the stability of Fréchet polynomials
}

Dan M. Dăianu*

This paper is dedicated to Professor Borislav Crstici for his 90th birthday

*Correspondence:

dan.daianu@mat.upt.ro

Department of Mathematics,

'Politehnica' University of Timişoara,

Piaţa Victoriei No. 2, Timişoara,

300006, Romania

\begin{abstract}
By means of a new stability result, established for symmetric and multi-additive mappings, and using the concepts of stability couple and of stability chain, we prove, by a recursive procedure, the generalized stability of two of Fréchet's polynomial equations. We also give a new functional characterization of generalized polynomials and a new approach to solving the generalized stability of the monomial equation.

MSC: 39B82; 39B52; 20M15; 65Q30
\end{abstract}

Keywords: multi-additive mapping; Fréchet polynomial; monomial; stability

\section{Motivation}

Two of the best known functional equations for which there are no satisfactory results as regards generalized stability in the sense of Bourgin [1] and Găvruța [2] are Fréchet's polynomial equations:

$$
D^{n} p\left(x_{1}, \ldots, x_{n+1}\right)=0, \quad x_{1}, \ldots, x_{n+1} \in M
$$

(see [3] for the original equation) and

$$
\Delta_{y}^{n+1} p(x)=0, \quad x, y \in M
$$

(see [4], for the original equation), where $p: M \rightarrow B$ is the unknown function, $M$ is an abelian monoid, $B$ is a real (or complex) Banach space, $n$ is a positive integer and, for any mapping $f: M \rightarrow B, D^{n-1} f: M^{n} \rightarrow B$ is the symmetric function defined by

$$
D^{n-1} f\left(x_{1}, \ldots, x_{n}\right):=\sum_{\epsilon_{1}, \ldots, \epsilon_{n}=0}^{1}(-1)^{n-\left(\epsilon_{1}+\cdots+\epsilon_{n}\right)} f\left(\epsilon_{1} x_{1}+\cdots+\epsilon_{n} x_{n}\right) .
$$

The Hyers-Ulam stability of equation (1.2) has been studied intensively. Starting with the fundamental work of Whitney [5, 6] and Hyers [7], in 1983 Albert and Baker [8] proved the Hyers-Ulam stability of equation (1.2) using the Hyers-Ulam stability of the equation

$$
\Delta_{x_{1}} \circ \cdots \circ \Delta_{x_{n+1}} p(x)=0, \quad x_{1}, \ldots, x_{n+1}, x \in M
$$

@2014 Dăianu; licensee Springer. This is an Open Access article distributed under the terms of the Creative Commons Attribution License (http://creativecommons.org/licenses/by/2.0), which permits unrestricted use, distribution, and reproduction in any medium, provided the original work is properly cited. 
which is equivalent to equation $(1.2)[9,10]$, but apparently more restrictive than equation (1.1). The only results of generalized stability in the sense of Bourgin and Găvruța for equation (1.2) have recently been obtained by Jun and Kim [11] and by Lee [12, 13] for $n \leq 3$.

The difficulties that arise in the proof of a criterion of the generalized stability for such equations with differences for an arbitrary $n$ are caused by the necessity of inventing a recursive procedure of determining the control functions and the monomial components of the approximated polynomial.

In this paper we eliminate these impediments with the help of two new instruments: the stability couples - which rigorously define the concept of the stability of a functional equation - and the stability chains - necessary for studying the stability of equation (1.1). First we prove - through the so-called direct method (of Hyers) - a new generalized stability theorem for multi-additive and symmetric functions in the spirit of Bourgin and Găvruța. We use this result to justify a recursive procedure of solving the stability of equation (1.1) and we prove the equivalence of equations (1.1), (1.2), and (1.4). We then give a general technique of solving the stability of equation (1.2) using the stability of equation (1.1). As a consequence, we obtain stability results of Hyers-Ulam type which extend and improve the above mentioned results, and Aoki-Rassias type stability results for equations (1.1) and (1.2). Finally, we give a new technique for proving the generalized stability of the monomial equation

$$
\Delta_{y}^{n} m(x)=(n !) m(y), \quad x, y \in M
$$

\section{Framework}

Throughout this paper we assume: $\mathbb{N}$ is the set of nonnegative integers, $n \geq 1$ is an integer, $M$ is an abelian monoid under addition, $B$ is a Banach space with the norm $\|\cdot\|$, and $B^{M}$ is the vector space of all functions from $M$ to $B$.

We recall some definitions and properties of difference operators that we use in the following sections (see for details [14] or [15]).

For $y \in M$, the linear operators $\Delta_{y}, \Delta_{y}^{0}: B^{M} \rightarrow B^{M}$ are defined by

$$
\Delta_{y} f(x):=f(x+y)-f(x), \quad \Delta_{y}^{0} f(x):=f(x) .
$$

For all $x, y \in M$ we have $\Delta_{x} \circ \Delta_{y}=\Delta_{y} \circ \Delta_{x}=\Delta_{x+y}-\Delta_{x}-\Delta_{y}$.

The $n$th iteration of $\Delta_{y}$, denoted $\Delta_{y}^{n}$, verifies the identity

$$
\Delta_{y}^{n} f(x)=\sum_{k=0}^{n}(-1)^{n-k}\left(\begin{array}{l}
n \\
k
\end{array}\right) f(x+k y), \quad x, y \in M, f \in B^{M} .
$$

Let $j \in \mathbb{N}$. The function $m: M \rightarrow B$ is a $j$-monomial if $\Delta_{y}^{j} m(x)=(j !) m(y)$ for all $x, y \in M$ (we agree that 0 ! $=1$ ). If $j \geq 1$, the $j$-monomial $m$ verifies the relation $m(k x)=k^{j} m(x), x \in M$, $k \in \mathbb{N}$.

We say that the mapping $p: M \rightarrow B$ is an $n$-polynomial if for any $j \in\{0, \ldots, n\}$ there exists a $j$-monomial $m_{j}: M \rightarrow B$ such that $p=m_{0}+m_{1}+\cdots+m_{n}$. 
If $g: M^{n} \rightarrow B$ is a symmetric function, we define $D_{n} g: M^{n+1} \rightarrow B$ by $D_{n} g\left(x_{1}, x_{2}\right):=g\left(x_{1}+\right.$ $\left.x_{2}\right)-g\left(x_{1}\right)-g\left(x_{2}\right)$, for $n=1$, and for $n>1$ by

$$
D_{n} g\left(x_{1}, \ldots, x_{n+1}\right):=g\left(x_{1}, \ldots, x_{n-1}, x_{n}+x_{n+1}\right)-g\left(x_{1}, \ldots, x_{n}\right)-g\left(x_{1}, \ldots, x_{n-1}, x_{n+1}\right) .
$$

The symmetric mapping $a: M^{n} \rightarrow B$ is n-additive if and only if

$$
D_{n} a(z)=0, \quad z \in M^{n+1} .
$$

The operator $D^{n-1}: B^{M} \rightarrow B^{M^{n}}$ defined in equation (1.3) can be described by the difference operators as follows:

$$
D^{n-1} f\left(x_{1}, \ldots, x_{n}\right):=\Delta_{x_{1}} \circ \cdots \circ \Delta_{x_{n}} f(0), \quad x_{1}, \ldots, x_{n} \in M, f \in B^{M} .
$$

Since $D^{1}=D_{1} \circ D^{0}$, and for $k>1$ and $x_{1}, \ldots, x_{k+1} \in M$ we have $\Delta_{x_{1}} \circ \cdots \circ \Delta_{x_{k-1}} \circ \Delta_{x_{k}} \circ$ $\Delta_{x_{k+1}}=\Delta_{x_{1}} \circ \cdots \circ \Delta_{x_{k-1}} \circ \Delta_{x_{k}+x_{k+1}}-\Delta_{x_{1}} \circ \cdots \circ \Delta_{x_{k-1}} \circ \Delta_{x_{k}}-\Delta_{x_{1}} \circ \cdots \circ \Delta_{x_{k-1}} \circ \Delta_{x_{k+1}}$, and it follows that

$$
D^{k}=D_{k} \circ D^{k-1}, \quad k=1,2, \ldots
$$

The following fundamental features of difference operators were given by Mazur and Orlicz $[9,10]$ on linear spaces and extended on commutative semigroups by Djoković [15].

Theorem 2.1 If $a: M^{n} \rightarrow B$ is a symmetric and n-additive mapping then the function $a^{*}: M \rightarrow B$ defined by $a^{*}(x):=a(x, \ldots, x)$ is an n-monomial,

$$
D^{n-1} a^{*}(y)=(n !) a(y), \quad y \in M^{n} \quad \text { and } \quad D^{n} a^{*}(z)=0, \quad z \in M^{n+1} .
$$

Theorem 2.2 The function $p: M \rightarrow B$ is an n-polynomial if and only if it verifies one of the equivalent equations (1.2) and (1.4). In these conditions, for any $i \in\{1, \ldots, n\}$ there is a symmetric and $i$-additive mapping $a_{i}: M^{i} \rightarrow B$ such that

$$
p=a_{0}^{*}+a_{1}^{*}+\cdots+a_{n}^{*}
$$

where $a_{0}^{*}: M \rightarrow B$ is the 0 -monomial defined by $a_{0}^{*}(x):=p(0)$.

The concept of Hyers-Ulam stability of a functional equation has surfaced as a consequence of the first answer given by Hyers (for Cauchy's equation on Banach spaces [16]) to a question posed by Ulam in 1940 about the stability of group morphisms. The concept was extended by Aoki [17], Bourgin [1], Rassias [18], Găvruță [2] and others. Here we consider that a functional equation is stable if it admits a nontrivial stability couple.

Definition 2.3 Let $E a(z)=0, z \in S^{n}$ be a functional equation, where $E: A \subseteq B^{S} \rightarrow B^{S^{n}}$ is a mapping, $S$ is a nonempty set and $a \in A$ is the unknown function. Let also $\varphi: S^{n} \rightarrow[0, \infty)$ and $\Phi: S \rightarrow[0, \infty)$ be two mappings. The pair $(\varphi, \Phi)$ is called a stability couple for the functional equation $E a=0$, if for all $f \in A$ for which

$$
\|E f(z)\| \leq \varphi(z), \quad z \in S^{n}
$$


there exists a function $a \in A$ such that $E a=0$ and

$$
\|f(x)-a(x)\| \leq \Phi(x), \quad x \in S .
$$

If, in addition, $a$ is the unique mapping with these properties, we say that $(\varphi, \Phi)$ is a strong stability couple.

If $(\varphi, \Phi)$ is a stability couple, $\varphi$ is called a control function. If for any constant and positive function $\varphi$ there is a stability couple $(\varphi, \Phi)$ for equation $E a=0$, we say that this equation is stable in the Hyers-Ulam sense. If $S$ is a normed vector space and there is a nontrivial stability couple $(\varphi, \Phi)$ (i.e. $\varphi \neq 0)$ such that the control function $\varphi$ is defined with the help of the norm from $S$, we say that the equation is stable in the Aoki-Rassias sense (see [17] and [18] for the origin of the eponymies).

We recall only two classic stability results, reformulated in terms of stability couples.

Theorem 2.4 [2] Let $G$ be an abelian group, $\varphi: G^{2} \rightarrow B$ a function such that

$$
\Psi(z):=\sum_{k=0}^{\infty} 2^{-(k+1)} \varphi\left(2^{k} z\right)<\infty, \quad z \in G^{2}
$$

and $\Phi(x):=\Psi(x, x)$. Then the pair $(\varphi, \Phi)$ is a strong stability couple for Cauchy's functional equation $\Delta_{y} a(x)-a(y)=0, x, y \in G$.

Theorem 2.5 [8] The functional equation (1.2) is stable in the Hyers-Ulam sense: if $M$ is an $(n+1)$ !-divisible abelian group and $\delta>0$, then there exists a positive constant $k_{n}$ such that $\left(\delta, k_{n} \delta\right)$ is a stability couple for equation (1.2).

The functional characterization of the real polynomial functions of degree less than or equal to $n$ with the continuous solutions of equation (1.1), or of equation (1.2) - seen as generalizations of Cauchy's equation - was realized by M. Fréchet in [3] and [4]. Fundamental studies of Fréchet's equations (1.2) and (1.4) on more general structures can be found in $[9,10,14,15,19]$. Some classical works on the stability of Cauchy's equation are $[1,2,16-18,20,21]$. The stability of some particular polynomials has been studied by a great number of authors [22]. Aoki-Rassias type theorems for equation (1.4) are given in [23] and [24]. For some results on stability of multi-additive mappings we refer the reader to $[8,25,26]$, on stability of monomials to $[8,27-33]$ and on stability of other different kinds of polynomials to $[7,8,26,34-44]$.

\section{Stability of symmetric and $\boldsymbol{n}$-additive mappings}

In the proving of the generalized stability - part of the existence - the following lemma is very useful.

Lemma 3.1 Let $\left(b_{k}\right)_{k \in \mathbb{N}}$ be a sequence in $B$, let $\left(\alpha_{k}\right)_{k \in \mathbb{N}}$ be a sequence in $[0, \infty)$ and $c>0$ such that $\beta:=\sum_{k=0}^{\infty} c^{-k-1} \alpha_{k}<\infty$. If $\left\|b_{k+1}-c b_{k}\right\| \leq \alpha_{k}$ for all $k \in \mathbb{N}$, then $\left(c^{-k} b_{k}\right)_{k \in \mathbb{N}}$ is a convergent sequence and $\left\|b-b_{0}\right\| \leq \beta$, where $b=\lim _{k \rightarrow \infty} c^{-k} b_{k}$. 
Proof According to the hypothesis it follows that

$$
\left\|c^{-k-1} b_{k+1}-c^{-k} b_{k}\right\| \leq c^{-k-1} \alpha_{k}, \quad k \in \mathbb{N} .
$$

Let $j$ be an arbitrary positive integer. Then, by using the triangle inequality and the above inequality, we obtain

$$
\left\|c^{-k-j} b_{k+j}-c^{-k} b_{k}\right\| \leq c^{-k-j} \alpha_{k+j-1}+c^{-k-j+1} \alpha_{k+j-2}+\cdots+c^{-k-1} \alpha_{k}
$$

hence

$$
\left\|c^{-k-j} b_{k+j}-c^{-k} b_{k}\right\| \leq \sum_{i=k}^{\infty} c^{-i-1} \alpha_{i}, \quad k \in \mathbb{N}
$$

Since $\lim _{k \rightarrow \infty} \sum_{i=k}^{\infty} c^{-i-1} \alpha_{i}=0$, it follows that $\left(c^{-k} b_{k}\right)_{k \in \mathbb{N}}$ is a Cauchy sequence. Let $b=: \lim _{k \rightarrow \infty} c^{-k} b_{k}$. Then, for $k=0$ and $j \rightarrow \infty$ in the previous inequality, we obtain $\left\|b-b_{0}\right\| \leq \beta$.

The following result is crucial in determining of strong stability couples for the functional equations (2.1), (1.1), (1.2), and (1.5).

Lemma 3.2 Let $K$ be a commutative semigroup, $r>0$ and let $\varphi: K \rightarrow[0, \infty)$ be a function such that $\widetilde{\varphi}(x):=\sum_{k=0}^{\infty} 2^{-r(k+1)} \varphi\left(2^{k} x\right)<\infty, x \in K$. Then

$$
\lim _{k \rightarrow \infty} 2^{-r k} \widetilde{\varphi}\left(2^{k} x\right)=0 \quad \text { and } \quad \sum_{k=0}^{\infty} 2^{-(r+1)(k+1)} \widetilde{\varphi}\left(2^{k} x\right) \leq 2^{-r} \widetilde{\varphi}(x), \quad x \in K .
$$

Proof For $x \in K$ we have

$$
\lim _{k \rightarrow \infty} 2^{-r k} \widetilde{\varphi}\left(2^{k} x\right)=\lim _{k \rightarrow \infty} \sum_{i=0}^{\infty} 2^{-r(k+i+1)} \varphi\left(2^{k+i} x\right)=\lim _{k \rightarrow \infty}\left[\widetilde{\varphi}(x)-\sum_{j=0}^{k-1} 2^{-r(j+1)} \varphi\left(2^{j} x\right)\right]=0,
$$

and also

$$
\begin{aligned}
\sum_{k=0}^{\infty} 2^{-(r+1)(k+1)} \widetilde{\varphi}\left(2^{k} x\right) & =\sum_{k=0}^{\infty} \sum_{j=0}^{\infty} 2^{-(r+1)(k+1)-r(j+1)} \varphi\left(2^{k+j} x\right) \\
& =2^{-r-1} \sum_{i=0}^{\infty} 2^{-r(i+1)} \sum_{k=0}^{i} 2^{-k} \varphi\left(2^{i} x\right) \\
& =2^{-r} \sum_{i=0}^{\infty}\left(1-2^{-i-1}\right) 2^{-r(i+1)} \varphi\left(2^{i} x\right) \leq 2^{-r} \widetilde{\varphi}(x) .
\end{aligned}
$$

The operators $r_{n}, R_{n}$, and the set $\mathcal{D}_{n}^{+}$defined in the following lines will play a key role in building concrete stability couples for equations (2.1), (1.1), (1.2), and (1.5). 
Let $\varphi: M^{n+1} \rightarrow[0, \infty)$ be a function. Then $r_{n} \varphi: M^{n} \rightarrow[0, \infty)$ is the mapping defined by $r_{1} \varphi\left(x_{1}\right):=\varphi\left(x_{1}, x_{1}\right)$ if $n=1$, and, for $n>1$, by

$$
\begin{aligned}
r_{n} \varphi\left(x_{1}, \ldots, x_{n}\right):= & \varphi\left(2 x_{1}, \ldots, 2 x_{n-1}, x_{n}, x_{n}\right)+2 \varphi\left(2 x_{1}, \ldots, 2 x_{n-2}, x_{n}, x_{n-1}, x_{n-1}\right)+\cdots \\
& +2^{n-2} \varphi\left(2 x_{1}, x_{n}, x_{n-1}, \ldots, x_{3}, x_{2}, x_{2}\right)+2^{n-1} \varphi\left(x_{n}, x_{n-1}, \ldots, x_{2}, x_{1}, x_{1}\right) .
\end{aligned}
$$

If $\widetilde{\varphi}(z):=\sum_{k=0}^{\infty} 2^{-n(k+1)} \varphi\left(2^{k} z\right)<\infty, z \in M^{n+1}$, then we define the mapping $R_{n} \varphi: M^{n} \rightarrow$ $[0, \infty)$ by

$$
R_{n} \varphi:=r_{n} \widetilde{\varphi}
$$

From Lemma 3.2 it follows that $\lim _{k \rightarrow \infty} 2^{-n k} R_{n} \varphi\left(2^{k} y\right)=0, y \in M^{n}$. Therefore

$$
\begin{aligned}
& \mathcal{D}_{n}^{+}:=\left\{\left(\varphi, \varphi^{\prime}\right) \mid \varphi: M^{n+1} \rightarrow[0, \infty), \sum_{k=0}^{\infty} 2^{-n(k+1)} \varphi\left(2^{k} z\right)<\infty, z \in M^{n+1},\right. \\
&\left.\varphi^{\prime}: M^{n} \rightarrow[0, \infty), \varphi^{\prime}(y) \geq R_{n} \varphi(y), \text { and } \lim _{k \rightarrow \infty} 2^{-n k} \varphi^{\prime}\left(2^{k} y\right)=0, y \in M^{n}\right\}
\end{aligned}
$$

is a nontrivial set.

Now, we are able to prove that $\mathcal{D}_{n}^{+}$is a set of strong stability couples for the functional equation (2.1), where the operator $D_{n}$ acts on the vector space of symmetric functions from $M^{n}$ to $B$. The following theorem extends Găvruță's result from Theorem 2.4.

Theorem 3.3 Let $\left(\varphi_{n+1}, \varphi_{n}\right) \in \mathcal{D}_{n}^{+}$and $g: M^{n} \rightarrow B$ be a symmetric function satisfying the inequality

$$
\left\|D_{n} g(z)\right\| \leq \varphi_{n+1}(z), \quad z \in M^{n+1} .
$$

Then $a(y):=\lim _{k \rightarrow \infty} 2^{-n k} g\left(2^{k} y\right)$ defines the unique symmetric and $n$-additive function $a$ : $M^{n} \rightarrow$ B such that

$$
\|g(y)-a(y)\| \leq \varphi_{n}(y), \quad y \in M^{n} .
$$

Moreover, $m:=a^{*}$ is the unique $n$-monomial from $B^{M}$ for which

$$
\|g(x, \ldots, x)-m(x)\| \leq \varphi_{n}(x, \ldots, x), \quad x \in M .
$$

Proof Let $y=\left(x_{1}, \ldots, x_{n}\right) \in M^{n}$. Putting $z=\left(x_{1}, \ldots, x_{n-1}, x_{n}, x_{n}\right)$ in (3.1) we get $\| D_{n} g\left(x_{1}, \ldots\right.$, $\left.x_{n-1}, x_{n}, x_{n}\right)\|=\| g\left(x_{1}, \ldots, x_{n-1}, 2 x_{n}\right)-2 g\left(x_{1}, \ldots, x_{n}\right) \|$, hence

$$
\left\|D_{n} g\left(x_{1}, \ldots, x_{n-1}, x_{n}, x_{n}\right)\right\| \leq \varphi_{n+1}\left(x_{1}, \ldots, x_{n-1}, x_{n}, x_{n}\right) .
$$

But $g$ is a symmetric function; therefore

$$
\begin{aligned}
& \left\|g(2 y)-2^{n} g(y)\right\| \\
& \quad=\|\left[g\left(2 x_{1}, 2 x_{2}, \ldots, 2 x_{n-1}, 2 x_{n}\right)-2 g\left(2 x_{1}, 2 x_{2}, \ldots, 2 x_{n-1}, x_{n}\right)\right]
\end{aligned}
$$




$$
\begin{aligned}
& +2\left[g\left(2 x_{1}, 2 x_{2}, \ldots, 2 x_{n-2}, x_{n}, 2 x_{n-1}\right)-2 g\left(2 x_{1}, 2 x_{2}, \ldots, 2 x_{n-2}, x_{n}, x_{n-1}\right)\right]+\cdots \\
& +2^{n-1}\left[g\left(x_{n}, x_{n-1}, \ldots, x_{2}, 2 x_{1}\right)-2 g\left(x_{n}, x_{n-1}, \ldots, x_{2}, x_{1}\right)\right] \| \\
\leq & \left\|D_{n} g\left(2 x_{1}, \ldots, 2 x_{n-1}, x_{n}, x_{n}\right)\right\|+2\left\|D_{n} g\left(2 x_{1}, \ldots, 2 x_{n-2}, x_{n}, x_{n-1}, x_{n-1}\right)\right\|+\cdots \\
& +2^{n-1}\left\|D_{n} g\left(x_{n}, x_{n-1}, \ldots, x_{2}, x_{1}, x_{1}\right)\right\| \\
\leq & r_{n} \varphi_{n+1}\left(x_{1}, \ldots, x_{n}\right),
\end{aligned}
$$

hence

$$
\left\|g(2 y)-2^{n} g(y)\right\| \leq r_{n} \varphi_{n+1}(y), \quad y \in M^{n} .
$$

Replacing $y$ by $2^{k} y$ we get

$$
\left\|g\left(2^{k+1} y\right)-2^{n} g\left(2^{k} y\right)\right\| \leq r_{n} \varphi_{n+1}\left(2^{k} y\right), \quad k \in \mathbb{N}, y \in M^{n} .
$$

Now, from Lemma 3.1 (for $b_{k}:=g\left(2^{k} y\right), c:=2^{n}, \alpha_{k}:=r_{n} \varphi_{n+1}\left(2^{k} y\right)$ and $\beta:=\varphi_{n}(y) \geq$ $\left.R_{n} \varphi_{n+1}(y)\right)$ it follows that $\left(2^{-n k} g\left(2^{k} y\right)\right)_{k \in \mathbb{N}}$ is a convergent sequence in the Banach space $B$, and its limit, $a(y)$, satisfies (3.2). But $g$ is symmetric, therefore $a$ is a symmetric function, too. From (3.1), the definition of the set $\mathcal{D}_{n}^{+}$, and Lemma 3.2 it follows that

$$
\lim _{k \rightarrow \infty} 2^{-n k}\left\|D_{n} g\left(2^{k} z\right)\right\| \leq \lim _{k \rightarrow \infty} 2^{-n k} \varphi_{n+1}\left(2^{k} z\right)=0,
$$

whence $D_{n} a(z)=0, z \in M^{n+1}$, i.e. $a$ is a symmetric and $n$-additive mapping that satisfies (3.2) and $a^{*}$ is a $n$-monomial that satisfies (3.3).

In order to justify the uniqueness of $a$, we consider the symmetric and $n$-additive mapping $a^{\prime}: M^{n} \rightarrow B$ for which $\left\|g(y)-a^{\prime}(y)\right\| \leq \varphi_{n}(y), y \in M^{n}$; since for any $y \in M^{n}$, $a^{\prime}\left(2^{k} y\right)=2^{n k} a^{\prime}(y)$, we have

$$
\lim _{k \rightarrow \infty}\left\|2^{-n k} g\left(2^{k} y\right)-a^{\prime}(y)\right\| \leq \lim _{k \rightarrow \infty} 2^{-n k} \varphi_{n}\left(2^{k} y\right)=0, \quad y \in M^{n},
$$

it follows that $a^{\prime}=a$.

Finally, if $m$ is an $n$-monomial that satisfies (3.3), that is, $\|g(x, \ldots, x)-m(x)\| \leq \varphi_{n}(x, \ldots, x)$, $x \in M$, since $m\left(2^{k} x\right)=2^{k n} m(x), x \in M$, it follows that

$$
\left\|2^{-n k} g\left(2^{k} x, \ldots, 2^{k} x\right)-m(x)\right\| \leq 2^{-n k} \varphi_{n}\left(2^{k} x, \ldots, 2^{k} x\right) \rightarrow 0,
$$

as $k \rightarrow \infty$; therefore $m=a^{*}$.

The following consequence is a stability result in the sense of Aoki-Rassias which generalizes Aoki's result from [17] and the result of Rassias from [18] (where the case $n=1$ was considered).

Corollary 3.4 Let $M$ be a linear normed space, $\delta>0, r \in(0,1)$ and let $\varphi_{n+1}: M^{n+1} \rightarrow$ $[0, \infty)$ be defined by $\varphi_{n+1}\left(x_{1}, x_{2}\right):=\delta\left(\left\|x_{1}\right\|^{r}+\left\|x_{2}\right\|^{r}\right)$ if $n=1$, and $\varphi_{n+1}\left(x_{1}, \ldots, x_{n+1}\right):=$ 
$\delta\left\|x_{1}\right\|^{r} \cdots\left\|x_{n-1}\right\|^{r}\left(\left\|x_{n}\right\|^{r}+\left\|x_{n+1}\right\|^{r}\right)$ if $n>1$. Suppose that $g: M^{n} \rightarrow B$ is a symmetric function such that

$$
\left\|D_{n} g(z)\right\| \leq \varphi_{n+1}(z), \quad z \in M^{n+1} .
$$

Then $a(y):=\lim _{k \rightarrow \infty} 2^{-n k} g\left(2^{k} y\right)$ defines the unique symmetric and $n$-additive mapping $a:$ $M^{n} \rightarrow$ B for which

$$
\left\|g\left(x_{1}, \ldots, x_{n}\right)-a\left(x_{1}, \ldots, x_{n}\right)\right\| \leq \frac{2 \delta}{2-2^{r}}\left\|x_{1}\right\|^{r} \cdots\left\|x_{n}\right\|^{r}, \quad x_{1}, \ldots, x_{n} \in M
$$

and $m:=a^{*}$ is the unique $n$-monomial that satisfies the inequality

$$
\|g(x, \ldots, x)-m(x)\| \leq \frac{2 \delta}{2-2^{r}}\|x\|^{n r}, \quad x \in M
$$

Proof It is sufficient to remark that $\left(\varphi_{n+1}, R_{n} \varphi_{n+1}\right) \in \mathcal{D}_{n}^{+}, r_{n} \varphi_{n+1}\left(x_{1}, \ldots, x_{n}\right)=\frac{2^{n}\left(1-2^{n(r-1)}\right)}{1-2^{r-1}} \times$ $\delta\left\|x_{1}\right\|^{r} \cdots\left\|x_{n}\right\|^{r}$, and $R_{n} \varphi_{n+1}=\frac{2^{-n}}{1-2^{n(r-1)}} r_{n} \varphi_{n+1}$.

\section{Stability of the equation $D^{n} p=0$}

The recurrence $D^{k}=D_{k} \circ D^{k-1}, k=1,2, \ldots$, is an essential tool in this section. First we complete Theorem 2.2.

Theorem 4.1 The function $p: M \rightarrow B$ is an n-polynomial if and only if $D^{n} p=0$.

Proof If $p$ is an $n$-polynomial then for any $j \in\{0, \ldots, n\}$ there exists a $j$-monomial $m_{j}: M \rightarrow$ $B$ such that $p=m_{0}+m_{1}+\cdots+m_{n}$. Then, for all $x, y \in M$ we have $\Delta_{y}^{j} m_{j}(x)=(j !) m_{j}(y)$, hence $\Delta_{y}^{j+1} m_{j}(x)=\Delta_{y}^{j} m_{j}(x+y)-\Delta_{y}^{j} m_{j}(x)=0, j \in\{0, \ldots, n\}$. Therefore $\Delta_{y}^{n+1} p(x)=0, x, y \in M$. From Theorem 2.2 it follows that $\Delta_{x_{1}} \circ \cdots \circ \Delta_{x_{n+1}} p(0)=0, x_{1}, \ldots, x_{n+1} \in M$, or, equivalently, $D^{n} p=0$.

Conversely, suppose that $D^{n} p=0$. Then

$$
\left[D_{n}\left(D^{n-1} p\right)\right](z)=0, \quad z \in M^{n+1}
$$

hence the symmetric function $D^{n-1} p$ is $n$-additive. From Theorem 2.1 it follows that $m_{n}:=$ $\left(D^{n-1} p\right)^{*}$ is an $n$-monomial and $D^{n-1} m_{n}=(n !) D^{n-1} p$. Therefore

$$
D^{n-1}\left(p-\frac{1}{n !} m_{n}\right)=0
$$

analogously (for $n>1), m_{n-1}:=\left(D^{n-1}\left(p-\frac{1}{n !} m_{n}\right)\right)^{*}$ is an $(n-1)$-monomial and

$$
D^{n-2}\left(p-\frac{1}{n !} m_{n}-\frac{1}{(n-1) !} m_{n-1}\right)=0 .
$$

By recurrence we finally obtain $D^{0}\left(p-\frac{1}{n !} m_{n}-\cdots-\frac{1}{1 !} m_{1}\right)=0$, where

$$
m_{j}=\left(D^{j}\left(p-\frac{1}{n !} m_{n}-\frac{1}{(n-1) !} m_{n-1}-\cdots-\frac{1}{(j+1) !} m_{j+1}\right)\right)^{*}
$$


is a $j$-monomial for $j=n-1, n-2, \ldots, 1$. But $D^{0}\left(p-\frac{1}{n !} m_{n}-\frac{1}{(n-1) !} m_{n-1}-\cdots-\frac{1}{1 !} m_{1}\right)(x)=$ $p(x)-\frac{1}{n !} m_{n}(x)-\frac{1}{(n-1) !} m_{n-1}(x)-\cdots-\frac{1}{1 !} m_{1}(x)-p(0)=0, x \in M$ and $m_{0}(x):=p(0)$ defines a 0 -monomial. Consequently $p=\sum_{j=0}^{n} \frac{1}{j !} m_{j}$ and, therefore, $p$ is an $n$-polynomial.

The central idea in justifying the fact that a pair $(\varphi, \Phi)$ is a stability couple for equation (1.1) is the existence of a stability chain $\left(\varphi_{n+1}, \varphi_{n}, \ldots, \varphi_{1}\right)$ between the mappings $\varphi$ and $\Phi$.

Definition 4.2 We say that $\left(\varphi_{n+1}, \varphi_{n}, \ldots, \varphi_{1}\right)$ is a stability chain between the functions $\varphi$ : $M^{n+1} \rightarrow[0, \infty)$ and $\Phi: M \rightarrow[0, \infty)$ if $\left(\varphi_{i+1}, \varphi_{i}\right)$ is a stability couple for the equation $D_{i} a=0$, $i \in\{1,2, \ldots, n\}, \varphi_{n+1}=\varphi$ on $M^{n+1}$, and $\varphi_{1}=\Phi$ on $M \backslash\{0\}$.

Remark Theorem 3.3 provides stability chains: if $\left(\varphi_{i+1}, \varphi_{i}\right) \in \mathcal{D}_{i}^{+}$for all $i \in\{1, \ldots, n\}$, then $\left(\varphi_{n+1}, \varphi_{n}, \ldots, \varphi_{1}\right)$ is a stability chain between the $\varphi_{n+1}$ and $\varphi_{1}$.

Stability chains provide stability couples for equation (1.1)

Theorem 4.3 If there exists a stability chain between $\varphi: M^{n+1} \rightarrow[0, \infty)$ and $\Phi: M \rightarrow$ $[0, \infty)$, then $(\varphi, \Phi)$ is a stability couple for equation (1.1).

Proof Let $\left(\varphi_{n+1}, \varphi_{n}, \ldots, \varphi_{1}\right)$ be a stability chain between the functions $\varphi=\varphi_{n+1}$ and $\Phi$. Let also $f: M \rightarrow B$ be a mapping such that $\left\|D^{n} f(z)\right\| \leq \varphi(z), z \in M^{n+1}$. Since $D^{n}=D_{n} \circ D^{n-1}$, we have

$$
\left\|\left[D_{n}\left(D^{n-1} f\right)\right](z)\right\| \leq \varphi_{n+1}(z), \quad z \in M^{n+1} .
$$

Because $\left(\varphi_{n+1}, \varphi_{n}\right)$ is a stability couple for equation (2.1) and $D^{n-1} f: M^{n} \rightarrow B$ is a symmetric mapping, it follows that there is a symmetric and $n$-additive mapping $a_{n}: M^{n} \rightarrow B$, such that $\left\|D^{n-1} f(y)-a_{n}(y)\right\| \leq \varphi_{n}(y), y \in M^{n}$. According to Theorem 2.1 we have $D^{n-1} a_{n}^{*}=(n !) a_{n}$, hence

$$
\left\|\left[D^{n-1}\left(f-\frac{1}{n !} a_{n}^{*}\right)\right](y)\right\| \leq \varphi_{n}(y), \quad y \in M^{n}
$$

By reverse induction we finally obtain

$$
\left\|\left[D^{0}\left(f-\frac{1}{n !} a_{n}^{*}-\frac{1}{(n-1) !} a_{n-1}^{*}-\cdots-a_{1}^{*}\right)\right](x)\right\| \leq \varphi_{1}(x), \quad x \in M,
$$

hence

$$
\left\|f(x)-f(0)-a_{1}^{*}(x)-\cdots-\frac{1}{n !} a_{n}^{*}(x)\right\| \leq \Phi(x), \quad x \in M
$$

where $a_{j}: M^{k} \rightarrow B$ is a symmetric and $j$-additive mapping, $j \in\{1, \ldots, n\}$. According to Theorem 2.1, $p:=f(0)+a_{1}^{*}+\cdots+\frac{1}{n !} a_{n}^{*}$ is an $n$-polynomial, and, from Theorem 4.2, it follows that $D^{n} p=0$. Consequently, $(\varphi, \Phi)$ is a stability couple for equation (1.1).

The following theorem provides a technique of building strong stability couples for equation (1.1) and is the main result of this section. 
Theorem 4.4 Suppose that $\left(\varphi_{i+1}, \varphi_{i}\right) \in D_{i}^{+}$for all $i \in\{1, \ldots, n\}, \Phi(x)=\varphi_{1}(x)$ for all $x \in M \backslash$ $\{0\}$, and $\Phi(0)=0$. If $: M \rightarrow B$ is a function satisfying

$$
\left\|D^{n} f(z)\right\| \leq \varphi_{n+1}(z), \quad z \in M^{n+1}
$$

then there exists a unique n-polynomial $p: M \rightarrow B$ such that

$$
\|f(x)-p(x)\| \leq \Phi(x), \quad x \in M
$$

Moreover, $p=: \sum_{j=0}^{n} \frac{1}{j !} m_{j}$, where $m_{j}$ is a $j$-monomial for any $j \in\{0, \ldots, n\}, m_{0}(x):=f(0)$, and the monomials $m_{j}, j \in\{1, \ldots, n\}$, can be obtained by recurrence: let $f_{n}:=f$; then, for $j=n, n-1, \ldots, 1$, we have the alternative

$$
\begin{aligned}
& m_{j}(x):=\lim _{k \rightarrow \infty} 2^{-k j} D^{j-1} f_{j}\left(2^{k} x, \ldots, 2^{k} x\right), \quad \text { or } \quad m_{j}(x):=(j !) \lim _{k \rightarrow \infty} 2^{-k j} f_{j}\left(2^{k} x\right), \\
& \quad \text { and } f_{j-1}:=f_{j}-\frac{1}{j !} m_{j} .
\end{aligned}
$$

Proof Since $\left(\varphi_{i+1}, \varphi_{i}\right) \in \mathcal{D}_{i}^{+}, i \in\{1, \ldots, n\}$, from Theorem 3.3 and Theorem 4.3 it follows that $\left(\varphi_{n+1}, \varphi_{n}, \ldots, \varphi_{1}\right)$ is a stability chain between $\varphi_{n+1}$ and $\Phi$, the pair $(\varphi, \Phi)$ is a stability couple for equation (1.1), and there exists an $n$-polynomial $p$ satisfying (4.2).

We successively apply Theorem 3.3 and Theorem 2.1 (as in the proof of Theorem 4.3) for justifying procedure (4.3). From (4.1) we have

$$
\left\|\left[D_{n}\left(D^{n-1} f_{n}\right)\right](z)\right\| \leq \varphi_{n+1}(z), \quad z \in M^{n+1} .
$$

Since $\left(\varphi_{n+1}, \varphi_{n}\right) \in \mathcal{D}_{n}^{+}$, from Theorem 3.3 it follows that

$$
m_{n}(x):=\lim _{k \rightarrow \infty} 2^{-k n} D^{n-1} f_{n}\left(2^{k} x, \ldots, 2^{k} x\right)
$$

defines an $n$-monomial satisfying the inequality

$$
\left\|D^{n-1} f_{n-1}(y)\right\| \leq \varphi_{n}(y), \quad y \in M^{n},
$$

where $f_{n-1}:=f_{n}-\frac{1}{n !} m_{n}$. Analogously, $\left(\varphi_{n}, \varphi_{n-1}\right) \in \mathcal{D}_{n-1}^{+}$(if $\left.n>1\right)$ and, from Theorem 3.3, it follows that

$$
m_{n-1}(x):=\lim _{k \rightarrow \infty} 2^{-k(n-1)} D^{n-2} f_{n-1}\left(2^{k} x, \ldots, 2^{k} x\right)
$$

defines an $(n-1)$-monomial satisfying the inequality

$$
\left\|D^{n-2} f_{n-2}(y)\right\| \leq \varphi_{n-1}(y), \quad y \in M^{n-1}
$$

where $f_{n-2}:=f_{n-1}-\frac{1}{(n-1) !} m_{n-1}$. By recurrence, we finally find that

$$
m_{1}(x):=\lim _{k \rightarrow \infty} 2^{-k} D^{0} f_{1}\left(2^{k} x\right)
$$


defines an additive function such that

$$
\left\|D^{0} f_{0}(x)\right\|=\left\|f_{0}(x)-f_{0}(0)\right\| \leq \varphi_{1}(x), \quad x \in M
$$

where $f_{0}:=f_{1}-m_{1}$. Since $f_{j-1}=f_{j}-\frac{1}{j !} m_{j}, j \in\{1, \ldots, n\}$ it follows that $f_{0}=f-\sum_{j=1}^{n} \frac{1}{j !} m_{j}$ and $f_{0}(0)=f(0)$. Therefore the above inequality becomes

$$
\|f(x)-p(x)\| \leq \varphi_{1}(x), \quad x \in M .
$$

From $\varphi_{1}(x)=\Phi(x)$ for $x \neq 0$ and $\Phi(0)=0$ it follows that (4.2) is satisfied for the $n$ polynomial $p=\sum_{j=0}^{n} \frac{1}{j !} m_{j}$, where $m_{j}(x)=\lim _{k \rightarrow \infty} 2^{-k j} D^{j-1} f_{j}\left(2^{k} x, \ldots, 2^{k} x\right), j \in\{1, \ldots, n\}$ and $m_{0}(x)=f(0), x \in M$.

We prove now by reverse induction that

$$
\left\|f_{j}(x)-\sum_{i=0}^{j} \frac{1}{i !} m_{i}(x)\right\| \leq \Phi(x), \quad \text { and } \quad m_{j}(x)=(j !) \lim _{k \rightarrow \infty} 2^{-k j} f_{j}\left(2^{k} x\right) \text {, }
$$

for $x \in M$ and $j=n, \ldots, 1$. Let $x \in M$. Since $m_{j}$ is a $j$-monomial and $\left(\varphi_{2}, \varphi_{1}\right) \in \mathcal{D}_{1}^{+}$we have

$$
m_{j}\left(2^{k} x\right)=2^{k j} m_{j}(x), \quad \text { and } \quad \lim _{k \rightarrow \infty} 2^{-j k} \Phi\left(2^{k} x\right)=0, \quad j=n, \ldots, 1 .
$$

We prove relations (4.4) for $j=n$. First, we remark that inequality (4.2) becomes

$$
\left\|f_{n}(x)-\sum_{i=0}^{n} \frac{1}{i !} m_{i}(x)\right\| \leq \Phi(x) .
$$

Replacing here $x$ by $2^{k} x$, multiplying both members of this inequality by $2^{-k n}$ and taking into account relations (4.5), we obtain

$$
\left\|2^{-k n} f_{n}\left(2^{k} x\right)-\sum_{i=0}^{n} 2^{-k(n-i)} \frac{1}{i !} m_{i}(x)\right\| \leq 2^{-k n} \Phi\left(2^{k} x\right) \rightarrow 0, \quad \text { as } k \rightarrow \infty,
$$

hence $\lim _{k \rightarrow \infty} 2^{-k n} f_{n}\left(2^{k} x\right)=\frac{1}{n !} m_{n}(x)$, and (4.4) is proved for $j=n$.

Since $f_{n-1}=f_{n}-\frac{1}{n !} m_{n},(4.2)$ becomes

$$
\left\|f_{n-1}(x)-\sum_{i=0}^{n-1} \frac{1}{i !} m_{i}(x)\right\| \leq \Phi(x) .
$$

By recurrence, we finally obtain $\left\|f_{1}(x)-m_{0}(x)-m_{1}(x)\right\| \leq \Phi(x)$, hence

$$
\left\|2^{-k} f_{1}\left(2^{k} x\right)-2^{-k} m_{0}(x)-m_{1}(x)\right\| \leq 2^{-k} \Phi\left(2^{k} x\right) \rightarrow 0, \quad \text { as } k \rightarrow \infty .
$$

Therefore $m_{1}(x)=\lim _{k \rightarrow \infty} 2^{-k} f_{1}\left(2^{k} x\right)$ and the alternative is completely proved.

We have yet to show that the only $n$-polynomial satisfying (4.2) is $p=\sum_{j=0}^{n} \frac{1}{j !} m_{j}$. Suppose that $m_{j}^{\prime}$ is a $j$-polynomial, $j \in\{0, \ldots, n\}$, and $p^{\prime}=\sum_{j=0}^{n} m_{j}^{\prime}$ verifies (4.2), i.e. $\left\|f(x)-p^{\prime}(x)\right\| \leq$ $\Phi(x), x \in M$. It follows immediately that

$$
\left\|p(x)-p^{\prime}(x)\right\| \leq 2 \Phi(x), \quad x \in M .
$$


Putting here $x=0$, we obtain $m_{0}=m_{0}^{\prime}$ and thus

$$
\left\|\sum_{j=1}^{n}\left[\frac{1}{j !} m_{j}(x)-m_{j}^{\prime}(x)\right]\right\| \leq 2 \Phi(x), \quad x \in M
$$

Again, replacing here $x$ by $2^{k} x$ and multiplying both members of this inequality by $2^{-k n}$, we obtain

$$
\left\|\frac{1}{n !} m_{n}(x)-m_{n}^{\prime}(x)-\sum_{j=1}^{n-1} 2^{-k(n-j)}\left[\frac{1}{j !} m_{j}(x)-m_{j}^{\prime}(x)\right]\right\| \leq 2^{-k n} \Phi\left(2^{k} x\right) \rightarrow 0
$$

as $k \rightarrow \infty$, namely $m_{n}^{\prime}=\frac{1}{n !} m_{n}$, and inequality $\left\|p(x)-p^{\prime}(x)\right\| \leq 2 \Phi(x)$ becomes

$$
\left\|\sum_{j=1}^{n-1}\left[\frac{1}{j !} m_{j}(x)-m_{j}^{\prime}(x)\right]\right\| \leq 2 \Phi(x)
$$

By reverse induction, we obtain $m_{j}^{\prime}=\frac{1}{j !} m_{j}$ for $j=n, n-1, \ldots, 1$. So $p^{\prime}=p$ and the theorem is proved.

Remark The condition $\Phi(0)=0$ imposed in the previous theorem is needed to ensure the uniqueness of the 0 -monomial $m_{0}$. In fact, as $D^{n} f(0)=0$, we can consider, without affecting the generality, $\varphi_{n+1}(0)=0$ in inequality (4.1).

The following consequence provides a class of strong stability couples for equation (1.1), and a technique for building stability chains.

Corollary 4.5 Let $\varphi: M^{n+1} \rightarrow[0, \infty)$ be a mapping such that $\sum_{k=0}^{\infty} 2^{-(k+1)} \varphi\left(2^{k} z\right)<\infty$, $z \in M^{n+1}$. Then for all functions $\Phi: M \rightarrow[0, \infty)$ for which

$$
\Phi(x) \geq R_{1} \cdots R_{n} \varphi(x), \quad \lim _{k \rightarrow \infty} 2^{-k} \Phi\left(2^{k} x\right)=0, \quad x \in M \backslash\{0\} \quad \text { and } \quad \Phi(0)=0,
$$

the pairs $(\varphi, \Phi)$ are strong stability couples for equation (1.1). Moreover, if $\varphi_{n+1}:=\varphi$ and $f$ : $M \rightarrow B$ is a function satisfying (4.1), then procedure (4.3) defines the unique n-polynomial $p$ that verifies (4.2).

Proof We successively apply Lemma 3.2. Let $\bar{\varphi}(z):=\sum_{k=0}^{\infty} 2^{-(k+1)} \varphi\left(2^{k} z\right)$. Then $\lim _{k \rightarrow \infty} 2^{-k} \bar{\varphi}\left(2^{k} z\right)=0$. Because $R_{n} \varphi(y) \leq r_{n} \bar{\varphi}(y), y \in M^{n}$, it follows that $\left(\varphi, R_{n} \varphi\right) \in \mathcal{D}_{n}^{+}$. By reverse induction, we obtain

$$
R_{j} R_{j+1} \cdots R_{n} \varphi(y) \leq r_{j} r_{j+1} \cdots r_{n} \bar{\varphi}(y), \quad \lim _{k \rightarrow \infty} 2^{-k} R_{j} \cdots R_{n} \varphi\left(2^{k} y\right)=0, \quad y \in M^{j}
$$

and

$$
\left(R_{j+1} \cdots R_{n} \varphi, R_{j} \cdots R_{n} \varphi\right) \in \mathcal{D}_{j}^{+}, \quad \text { for } j=n-1, n-2, \ldots, 1 .
$$

Since $\left(R_{2} \cdots R_{n} \varphi, R_{1} \cdots R_{n} \varphi\right) \in \mathcal{D}_{1}^{+}$it follows immediately that $\left(R_{2} \cdots R_{n} \varphi, \Phi\right) \in \mathcal{D}_{1}^{+}$and from Theorem 4.4, we obtain the conclusion. 
The following consequence is a stability result for equation (1.1) in the sense of HyersUlam.

Corollary 4.6 Let $\delta>0$. Iff $: M \rightarrow B$ is a function such that

$$
\left\|D^{n} f(z)\right\| \leq \delta, \quad z \in M^{n+1},
$$

then procedure (4.3) gives the unique n-polynomial p satisfying the condition

$$
\|f(x)-p(x)\| \leq \Phi(x)
$$

where $\Phi(x):=\delta$, for $x \in M \backslash\{0\}$ and $\Phi(0):=0$.

Proof Let $\varphi(z)=\delta, z \in M^{n+1}$. Then $r_{n} \varphi(y)=\left(2^{n}-1\right) \delta$, and $R_{n} \varphi(y)=\delta, y \in M^{n}$. By recurrence we obtain $R_{1} \cdots R_{n} \varphi=\delta$, and, from the previous corollary, we obtain the conclusion.

The functional equation (1.1) is stable in the Aoki-Rassias sense, as can be seen from the following corollary.

Corollary 4.7 Let $M$ be a normed space, $r \in(0,1)$ and $\delta>0$. If $\in B^{M}$ and

$$
\left\|D^{n} f\left(x_{1}, \ldots, x_{n+1}\right)\right\| \leq \delta\left(\left\|x_{1}\right\|^{r}+\cdots+\left\|x_{n+1}\right\|^{r}\right), \quad x_{1}, \ldots, x_{n+1} \in M,
$$

then there exists a unique n-polynomial $p=\sum_{j=0}^{n} \frac{1}{j !} m_{j}$ such that

$$
\|f(x)-p(x)\| \leq 2^{n} \delta\left(\prod_{i=1}^{n} \frac{2^{i}-1}{2^{i}-2^{r}}\right)\|x\|^{r}, \quad x \in M .
$$

The $j$-monomial $m_{j}, j \in\{1, \ldots, n\}$ is given in (4.3), and $m_{0}(x)=f(0), x \in M$.

Proof Let $\varphi\left(x_{1}, \ldots, x_{n+1}\right):=\delta\left(\left\|x_{1}\right\|^{r}+\cdots+\left\|x_{n+1}\right\|^{r}\right)$ and $\Phi(x):=2^{n} \delta\left(\prod_{i=1}^{n} \frac{2^{i}-1}{2^{i}-2^{r}}\right)\|x\|^{r}$. According to Corollary 4.5 , it is sufficient to show that $R_{1} \cdots R_{n} \varphi(x) \leq \Phi(x), x \in M$. It is straightforward to verify that

$$
r_{n} \varphi\left(x_{1}, \ldots, x_{n}\right) \leq 2 \delta\left(2^{n}-1\right)\left(\left\|x_{1}\right\|^{r}+\cdots+\left\|x_{n}\right\|^{r}\right) .
$$

Therefore

$$
R_{n} \varphi\left(x_{1}, \ldots, x_{n}\right) \leq 2 \delta \frac{2^{n}-1}{2^{n}-2^{r}}\left(\left\|x_{1}\right\|^{r}+\cdots+\left\|x_{n}\right\|^{r}\right), \quad x_{1}, \ldots, x_{n} \in M
$$

and, by recurrence, we finally obtain $R_{1} \cdots R_{n} \varphi(x) \leq \Phi(x), x \in M$.

\section{Stability of the equation $\Delta^{n+1} p=0$}

Further, we use the following conventions:

- $M$ is a uniquely $(n+1)$ !-divisible and commutative group. If $y \in M$ and $k \in\{1, \ldots, n+1\}$ we denote by $\frac{1}{k} y$ the unique solution of the equation $k x=y$. 
- Let $s \in\{0, \ldots, n+1\}$, and let $\pi:\{1, \ldots, n+1\} \rightarrow\{0, \ldots, n+1\} \backslash\{s\}$ be a bijection. For any $z=\left(z_{1}, \ldots, z_{n+1}\right) \in M^{n+1}$, the linear system

$$
x_{j}+\pi(j) y_{j}=0, \quad x_{j}+s y_{j}=z_{j}, \quad \text { for } j \in\{1, \ldots, n+1\}
$$

with the unknowns $x=\left(x_{1}, \ldots, x_{n+1}\right), y=\left(y_{1}, \ldots, y_{n+1}\right) \in M^{n+1}$ has a unique solution denoted by $x(z), y(z)$.

- If $\psi: M \times M \rightarrow[0, \infty)$ is a function, $s \in\{0, \ldots, n+1\}$, and $\pi:\{1, \ldots, n+1\} \rightarrow\{0, \ldots, n+1\} \backslash\{s\}$ is a bijection, then $\bar{\psi}: M^{n+1} \times M^{n+1} \rightarrow[0, \infty)$ is the mapping defined by

$$
\bar{\psi}\left(\left(x_{1}, \ldots, x_{n+1}\right),\left(y_{1}, \ldots, y_{n+1}\right)\right):=\sum_{\epsilon_{1}, \ldots, \epsilon_{n+1}=0}^{1} \psi\left(\epsilon_{1} x_{1}+\cdots+\epsilon_{n+1} x_{n+1}, \epsilon_{1} y_{1}+\cdots+\epsilon_{n+1} y_{n+1}\right)
$$

and $\psi_{\pi}^{s}: M^{n+1} \rightarrow[0, \infty)$ is the function defined by

$$
\psi_{\pi}^{s}(z):=\frac{1}{\left(\begin{array}{c}
n+1 \\
s
\end{array}\right)} \bar{\psi}(x(z), y(z))
$$

The following lemma establishes a fundamental connection between the behavior of the operators $\Delta^{n+1}$ and $D^{n}$.

Lemma 5.1 Let $s \in\{0, \ldots, n+1\}, \pi:\{1, \ldots, n+1\} \rightarrow\{0, \ldots, n+1\} \backslash\{s\}$ be a bijection, let $\psi: M \times M \rightarrow[0, \infty)$ be a mapping and let $f: M \rightarrow B$ be a function satisfying

$$
\left\|\Delta_{y}^{n+1} f(x)\right\| \leq \psi(x, y), \quad x, y \in M
$$

Then

$$
\left\|D^{n} f(z)\right\| \leq \psi_{\pi}^{s}(z), \quad z \in M^{n+1}
$$

Proof The central idea of this proof is to work with the operator $\Delta^{n+1}$ in the direct product $M^{n+1}$. Let $x=\left(x_{1}, \ldots, x_{n+1}\right), y=\left(y_{1}, \ldots, y_{n+1}\right) \in M^{n+1}$. Then

$$
\begin{aligned}
\left(\Delta_{y}^{n+1} D^{n} f\right)(x) & \\
= & \sum_{j=0}^{n+1}(-1)^{n+1-j}\left(\begin{array}{c}
n+1 \\
j
\end{array}\right) D^{n} f(x+j y) \\
= & \sum_{j=0}^{n+1}(-1)^{n+1-j}\left(\begin{array}{c}
n+1 \\
j
\end{array}\right) \\
& \cdot \sum_{\epsilon_{1}, \ldots, \epsilon_{n+1}=0}^{1}(-1)^{n+1-\left(\epsilon_{1}+\cdots+\epsilon_{n+1}\right)} f\left(\epsilon_{1} x_{1}+\cdots+\epsilon_{n+1} x_{n+1}+j\left(\epsilon_{1} y_{1}+\cdots+\epsilon_{n+1} y_{n+1}\right)\right) \\
= & \sum_{\epsilon_{1}, \ldots, \epsilon_{n+1}=0}^{1}(-1)^{n+1-\left(\epsilon_{1}+\cdots+\epsilon_{n+1}\right)} \Delta_{\epsilon_{1} y_{1}+\cdots+\epsilon_{n+1} y_{n+1}}^{n+1} f\left(\epsilon_{1} x_{1}+\cdots+\epsilon_{n+1} x_{n+1}\right) .
\end{aligned}
$$


From this, using the triangle inequality, hypothesis (5.2), and the definition of $\bar{\psi}$, we obtain $\left\|\left(\Delta_{y}^{n+1} D^{n} f\right)(x)\right\| \leq \bar{\psi}(x, y), x, y \in M^{n+1}$, or, equivalently,

$$
\left\|\sum_{j=0}^{n+1}(-1)^{n+1-j}\left(\begin{array}{c}
n+1 \\
j
\end{array}\right) D^{n} f(x+j y)\right\| \leq \bar{\psi}(x, y), \quad x, y \in M^{n+1} .
$$

Since $D^{n} f\left(x_{1}, \ldots, x_{n}, 0\right)=\Delta_{x_{1}} \circ \cdots \circ \Delta_{x_{n}}(f(0)-f(0))=0$ for all $x_{1}, \ldots, x_{n} \in M$, it follows immediately that, for all $z \in M^{n+1}$, we have

$$
D^{n} f(x(z)+s y(z))=D^{n} f(z) \text { and } \quad D^{n} f(x(z)+\pi(j) y(z))=0
$$

for all $j \in\{1, \ldots, n+1\}$, where $x(z), y(z)$ is the solution of system (5.1); consequently, for $x=x(z)$ and $y=y(z)$ in the previous inequality, we obtain $\left\|D^{n} f(z)\right\| \leq \psi_{\pi}^{s}(z), z \in M^{n+1}$.

The stability couples of equation (1.1) provide stability couples for equation (1.2): from Theorem 4.3, Theorem 4.4, and the previous lemma we obtain the following stability result.

Theorem 5.2 Let $s \in\{0, \ldots, n+1\}, \pi:\{1, \ldots, n+1\} \rightarrow\{0, \ldots, n+1\} \backslash\{s\}$ be a bijection, let $\psi: M \times M \rightarrow[0, \infty)$ be a mapping, and let $\left(\varphi_{n+1}, \Phi\right)$ be a (strong) stability couple for equation (1.1) such that

$$
\psi_{\pi}^{s}(z) \leq \varphi_{n+1}(z), \quad z \in M^{n+1} .
$$

Then $(\psi, \Phi)$ is a (strong) stability couple for equation (1.2). If, in addition, the pair $\left(\varphi_{n+1}, \Phi\right)$ verifies the conditions of Theorem 4.4 and $f: M \rightarrow B$ is a mapping that satisfies (5.2), then procedure (4.3) gives the unique n-polynomial p that verifies (4.2).

The consequences of Theorem 4.4 and the previous theorem provide specific classes of strong stability couples for Fréchet's second functional equation as follows.

Corollary 5.3 Let $s \in\{0, \ldots, n+1\}, \pi:\{1, \ldots, n+1\} \rightarrow\{0, \ldots, n+1\} \backslash\{s\}$ be a bijection, let $\psi: M \times M \rightarrow[0, \infty)$ be a function such that

$$
\sum_{k=0}^{\infty} 2^{-k} \psi\left(2^{k} x, 2^{k} y\right)<\infty, \quad x, y \in M
$$

and let $\Phi: M \rightarrow[0, \infty)$ be a mapping satisfying the conditions

$$
\Phi(0)=0, \quad \Phi(x) \geq R_{1} \cdots R_{n} \psi_{\pi}^{s}(x) \quad \text { and } \quad \lim _{k \rightarrow \infty} 2^{-k} \Phi\left(2^{k} x\right)=0
$$

for all $x \in M$. If $f: M \rightarrow B$ satisfies (5.2), then there exists a unique n-polynomial $p$ for which

$$
\|f(x)-p(x)\| \leq \Phi(x), \quad x \in M
$$

and the monomial components of $p$ can be calculated with procedure (4.3). 
Proof It is sufficient to note that $\sum_{k=0}^{\infty} 2^{-k} \psi_{\pi}^{s}\left(2^{k} z\right)<\infty, z \in M^{n+1}$, and that we can apply Corollary 4.5 for $\varphi=\psi_{\pi}^{s}$.

Applying Corollary 4.6, we obtain an improvement of Theorem 2.5.

Corollary 5.4 If $\epsilon>0$ and $f: M \rightarrow B$ verifies the inequality

$$
\left\|\Delta_{y}^{n+1} f(x)\right\| \leq \epsilon, \quad x, y \in M
$$

then there exists a unique n-polynomial p that satisfies the inequality

$$
\|f(x)-p(x)\| \leq \Phi(x), \quad x \in M
$$

where $\Phi(x)=\epsilon 2^{n+1} / \sup \left(\begin{array}{c}n+1 \\ j\end{array}\right)$, for $x \in M \backslash\{0\}$ and $\Phi(0)=0$. The monomial components of $p$ can be calculated with procedure (4.3).

Proof Let $s=[n / 2]+1$ and $\pi:\{1, \ldots, n+1\} \rightarrow\{0, \ldots, n+1\} \backslash\{s\}$ defined by $\pi(j)=j$ if $j \in$ $\{1, \ldots, n+1\} \backslash\{s\}$ and $\pi(s)=0$. Then $\left(\begin{array}{c}n+1 \\ s\end{array}\right)=\sup \left(\begin{array}{c}n+1 \\ j\end{array}\right)$. Defining $\psi(x, y):=\epsilon$, we have $\bar{\psi}(x, y)=$ $\epsilon\left[1+\left(\begin{array}{c}n+1 \\ 1\end{array}\right)+\cdots+\left(\begin{array}{c}n+1 \\ n+1\end{array}\right)\right]=\epsilon 2^{n+1}, x, y \in M^{n+1}$, and $\psi_{\pi}^{s}(z)=\epsilon 2^{n+1} / \sup \left(\begin{array}{c}n+1 \\ j\end{array}\right), z \in M^{n+1}$. From Corollary 4.6 it follows that $\left(\psi_{\pi}^{s}, \Phi\right)$ is a strong stability couple for equation (1.1); hence, from Theorem 5.2, it follows that $(\psi, \Phi)$ is a strong stability couple for equation (1.2) and that procedure (4.3) can be applied in this case.

The flexibility of working with stability couples is illustrated by the following AokiRassias type result.

Corollary 5.5 Let $M$ be a rational and normed vector space, $r \in(0,1)$, and $\epsilon>0$. If $f$ : $M \rightarrow B$ is a function satisfying

$$
\left\|\Delta_{y}^{n+1} f(x)\right\| \leq \epsilon\left(\|x\|^{r}+\|y\|^{r}\right), \quad x, y \in M
$$

then procedure (4.3) defines the unique n-polynomial $p: M \rightarrow$ B for which

$$
\|f(x)-p(x)\| \leq 4^{n+1} \epsilon\left(\prod_{i=1}^{n} \frac{2^{i}-1}{2^{i}-2^{r}}\right)\|x\|^{r}, \quad x \in M .
$$

Proof Let $s=0, \pi(j)=j, j \in\{1, \ldots, n+1\}$ and $\psi(x, y):=\epsilon\left(\|x\|^{r}+\|y\|^{r}\right)$. Then $\bar{\psi}\left(\left(x_{1}, \ldots, x_{n+1}\right)\right.$, $\left.\left(y_{1}, \ldots, y_{n+1}\right)\right) \leq 2^{n+1} \epsilon\left[\left(\left\|x_{1}\right\|^{r}+\cdots+\left\|x_{n+1}\right\|^{r}\right)+\left(\left\|y_{1}\right\|^{r}+\cdots+\left\|y_{n+1}\right\|\right)^{r}\right]$ for all $x_{1}, \ldots, x_{n+1}, y_{1}, \ldots$, $y_{n+1} \in M$. Let $z=\left(z_{1}, \ldots, z_{n+1}\right) \in M^{n+1}$. The system (5.1) has the solution

$$
x(z)=z, \quad y(z)=\left(-z_{1},-\frac{1}{2} z_{2}, \ldots,-\frac{1}{n} z_{n},-\frac{1}{n+1} z_{n+1}\right) .
$$

Therefore,

$$
\psi_{\pi}^{s}\left(z_{1}, \ldots, z_{n+1}\right) \leq 2^{n+2} \epsilon\left(\left\|z_{1}\right\|^{r}+\cdots+\left\|z_{n+1}\right\|\right)^{r}
$$


Applying Theorem 5.2 for $\varphi_{n+1}(z)=: 2^{n+2} \epsilon\left(\left\|z_{1}\right\|^{r}+\cdots+\left\|z_{n+1}\right\|\right)^{r}$, and Corollary 4.7 for $\delta=$ $2^{n+2} \epsilon$, it follows that procedure (4.3) gives the unique $n$-polynomial $p$ that satisfies (5.3).

Finally, we give a stability theorem for the monomial equation (1.5) (see [8, 27-33] for other approaches).

Theorem 5.6 Let $s \in\{0, \ldots, n+1\}, \pi:\{1, \ldots, n+1\} \rightarrow\{0, \ldots, n+1\} \backslash\{s\}$ be a bijection, let $\psi: M \times M \rightarrow[0, \infty)$ be a function so that

$$
\sum_{k=0}^{\infty} 2^{-k n} \psi\left(2^{k} x, 2^{k} y\right)<\infty, \quad x, y \in M
$$

and $\widetilde{\psi}(x, y):=\psi(x+y, y)+\psi(x, y)$. If $g: M \rightarrow B$ is a mapping such that

$$
\left\|\Delta_{y}^{n} g(x)-(n !) g(y)\right\| \leq \psi(x, y), \quad x, y \in M
$$

then there exists a unique n-monomial $m: M \rightarrow B$ that verifies the inequality

$$
\|g(y)-m(y)\| \leq \frac{1}{n !}\left[\psi(0, y)+R_{n} \widetilde{\psi}_{\pi}^{s}(y, \ldots, y)\right], \quad y \in M
$$

The $n$-monomial $m$ is given by $m(y):=\lim _{k \rightarrow \infty} 2^{-k n} g\left(2^{k} y\right)$.

Proof Let us first note that from $\sum_{k=0}^{\infty} 2^{-k n} \psi\left(2^{k} x, 2^{k} y\right)<\infty, x, y \in M$ it follows that

$$
\sum_{k=0}^{\infty} 2^{-k n} \widetilde{\psi}_{\pi}^{s}\left(2^{k} z\right)<\infty, \quad z \in M^{n+1}
$$

and from Lemma 3.2 it follows that $\left(\widetilde{\psi}_{\pi}^{s}, R_{n} \widetilde{\psi}_{\pi}^{s}\right) \in \mathcal{D}_{n}^{+}$. For all $x, y \in M$ we have $\left\|\Delta_{y}^{n+1} g(x)\right\| \leq$ $\left\|\Delta_{y}^{n} g(x+y)-(n !) g(y)-\left(\Delta_{y}^{n} g(x)-(n !) g(y)\right)\right\| \leq \widetilde{\psi}(x, y)$; hence, from Lemma 5.1 we have $\left\|D^{n} g(z)\right\| \leq \widetilde{\psi}_{\pi}^{s}(z), z \in M^{n+1}$, or, equivalently,

$$
\left\|\left[D_{n}\left(D^{n-1} g\right)\right](z)\right\| \leq \widetilde{\psi}_{\pi}^{s}(z), \quad z \in M^{n+1} .
$$

From Theorem 3.3, it follows that there exists a unique $n$-additive mapping $a: M^{n} \rightarrow B$ so that $\left\|D^{n-1} g(y, \ldots, y)-a^{*}(y)\right\| \leq R_{n} \widetilde{\psi}_{\pi}^{s}(y, \ldots, y), y \in M$, or, equivalently,

$$
\left\|\Delta_{y}^{n} g(0)-a^{*}(y)\right\| \leq R_{n} \widetilde{\psi}_{\pi}^{s}(y, \ldots, y), \quad y \in M
$$

Let $m=\frac{1}{n !} a^{*}$. Then

$$
\|g(y)-m(y)\| \leq\left\|g(y)-\frac{1}{n !} \Delta_{y}^{n} g(0)\right\|+\frac{1}{n !}\left\|\Delta_{y}^{n} g(0)-a^{*}(y)\right\|,
$$

and, from (5.4) and (5.6), it follows that the $n$-monomial $m$ satisfies (5.5). But $m\left(2^{k} y\right)=$ $2^{n k} m(y)$; therefore, from (5.5) we have for all $y \in M$

$$
\left\|2^{-k n} g\left(2^{k} y\right)-m(y)\right\| \leq \frac{1}{n !}\left[2^{-k n} \psi\left(0,2^{k} y\right)+2^{-k n} R_{n} \widetilde{\psi}_{\pi}^{s}\left(2^{k} y, \ldots, 2^{k} y\right)\right]
$$


Letting $k \rightarrow \infty$ in (5.7) and taking into account that $\left(\widetilde{\psi}_{\pi}^{s}, R_{n} \widetilde{\psi}_{\pi}^{s}\right) \in \mathcal{D}_{n}^{+}$, we obtain $m(y)=$ $\lim _{k \rightarrow \infty} 2^{-k n} g\left(2^{k} y\right), y \in M$.

Finally, if $m^{\prime}$ is an $n$-monomial that verifies (5.5), then $\left\|m(y)-m^{\prime}(y)\right\| \leq\|m(y)-g(y)\|+$ $\left\|g(y)-m^{\prime}(y)\right\| \leq 2 \frac{1}{n !}\left[\psi(0, y)+R_{n} \widetilde{\psi}_{\pi}^{s}(y, \ldots, y)\right]$. Since $m^{\prime}\left(2^{k} y\right)=2^{n k} m^{\prime}(y)$, we have

$$
\left\|m(y)-m^{\prime}(y)\right\| \leq 2 \frac{1}{n !}\left[2^{-k n} \psi\left(0,2^{k} y\right)+2^{-k n} R_{n} \widetilde{\psi}_{\pi}^{s}\left(2^{k} y, \ldots, 2^{k} y\right)\right] \rightarrow 0
$$

as $k \rightarrow \infty$. Therefore, $m$ is the only $n$-monomial that satisfies (5.5).

\section{Future work}

As future work we propose two unsolved problems.

1. Suppose that $\left(\varphi_{i+1}, \varphi_{i}\right) \in \mathcal{D}_{i}^{+}$for all $i \in\{1, \ldots, n\}, \alpha: M \rightarrow[0, \infty)$ is an arbitrary function, $\varphi_{n+1}^{\prime}\left(x_{1}, \ldots, x_{n+1}, x\right):=\alpha(x) \varphi_{n+1}\left(x_{1}, \ldots, x_{n+1}\right), \Phi(x):=\alpha(0) \varphi_{1}(x)$ for all $x \in M \backslash\{0\}$, and $\Phi(0)=0$. Then $\left(\varphi_{n+1}^{\prime}, \Phi\right)$ is a strong stability couple for equation (1.4) (see Theorem 4.4).

2. New stability couples for equations (2.1), (1.1), (1.2), and (1.5) can be determined using the ideas of the above theory, but replacing the operator $R_{n}$ with the operator $R_{n}^{-}$ defined by $R_{n}^{-} \varphi:=r_{n} \bar{\varphi}$, where $\varphi: M^{n+1} \rightarrow[0, \infty)$ is a function for which $\bar{\varphi}(z):=\sum_{k=0}^{\infty} 2^{n k} \varphi_{n+1}\left(2^{-k-1} z\right)<\infty, z \in M^{n+1}$, and $M$ is a commutative 2-divisible monoid (see also [20]).

The main results of this research paper are:

- the first proofs of generalized stability for two of the best known functional equations: the Fréchet polynomial equations;

- a proof of the equivalence of these two equations;

- a very general iterative technique for solving the stability of polynomial equations that can be applied to other similar problems;

- extensions and improvements of some known results of Hyers-Ulam type;

- a new technique for proving the generalized stability of the monomial equation.

\section{Competing interests}

The author declares that he has no competing interests.

\section{Acknowledgements}

I would like to thank to the referees for careful reading of this paper and their useful comments.

Received: 4 March 2013 Accepted: 17 December 2013 Published: 15 Jan 2014

\section{References}

1. Bourgin, D: Classes of transformations and bordering transformations. Bull. Am. Math. Soc. 57, 223-237 (1951)

2. Găvruţă, P: A generalization of the Hyers-Ulam-Rassias stability of approximately additive mappings. J. Math. Anal. Appl. 184, 431-438 (1994)

3. Fréchet, M: Une definition fonctionnelle des polynômes. Nouv. Ann. Math. 9, 145-182 (1909)

4. Fréchet, M: Les polynômes abstraits. J. Math. Pures Appl. 8, 71-92 (1929)

5. Whitney, H: On functions with bounded $n$th differences. J. Math. Pures Appl. 36(9), 87-95 (1957)

6. Whitney, H: On bounded functions with bounded nth differences. Proc. Am. Math. Soc. 10, 480-481 (1959)

7. Hyers, D: Transformations with bounded $m$-th differences. Pac. J. Math. 11, 591-602 (1961)

8. Albert, M, Baker, J: Functions with bounded $n$th differences. Ann. Pol. Math. 43, 93-103 (1983)

9. Mazur, S, Orlicz, W: Grundlegende Eigenschaften der polynomischen Operationen, I. Stud. Math. 5, 50-88 (1934)

10. Mazur, S, Orlicz, W: Grundlegende Eigenschaften der polynomischen Operationen, II. Stud. Math. 5, 179-189 (1934)

11. Jun, KW, Kim, HM: On the Hyers-Ulam-Rassias stability of a general cubic functional equation. Math. Inequal. Appl. 8(2), 289-302 (2003)

12. Lee, YH: On the generalized Hyers-Ulam stability of the generalized polynomial function of degree 3. Tamsui Oxford Univ. J. Math. Sci. 24(4), 429-444 (2008)

13. Lee, YH: On the Hyers-Ulam-Rassias stability of the generalized polynomial function of degree 2. J. Chungcheong Math. Soc. 22(2), 201-210 (2009) 
14. Hyers, D: Polynomial operators. In: Rassias, T (ed.) Topics in Mathematical Analysis, pp. 410-444. World Scientific, Teaneck (1989)

15. Djoković, D: A representation theorem for $\left(X_{1}-1\right)\left(X_{2}-1\right) \cdots\left(X_{n}-1\right)$ and its applications. Ann. Pol. Math. 22, 189-198 (1969)

16. Hyers, D: On the stability of the linear functional equation. Proc. Natl. Acad. Sci. USA 27, $222-224$ (1941)

17. Aoki, T: On the stability of the linear transformation in Banach spaces. J. Math. Soc. Jpn. 2, 84-88 (1950)

18. Rassias, T: On the stability of the linear mapping in Banach spaces. Proc. Am. Math. Soc. 72, 297-300 (1978)

19. Lijn, G: Les polynômes abstraits. Bull. Sci. Math. 84, 55-80, 102-112, 183-198 (1940)

20. Gajda, Z: On stability of additive mappings. Int. J. Math. Sci. 14, 431-434 (1991)

21. Rassias, T, Šemrl, P: On the behavior of mappings which do not satisfy Hyers-Ulam stability. Proc. Am. Math. Soc. 114 , 989-993 (1992)

22. Jung, SM: Hyers-Ulam-Rassias Stability of Functional Equations in Nonlinear Analysis. Springer Optimization and Its Applications, vol. 48. Springer, New York (2011)

23. Borelli, C, Invernizzi, C: Sulla stabilitá dell'equazione funzionale dei polinomi. Rend. Semin. Mat. (Torino) 57(3), 197-208 (1999)

24. Kaiser, Z: Stability of functional equations in abstract structures. PhD thesis, University of Debrecen (2005)

25. Ciepliński, K: Generalized stability of multi-additive mappings. Appl. Math. Lett. 23, 1291-1294 (2010)

26. Gajda, Z: Local stability of the functional equation characterizing polynomial functions. Ann. Pol. Math. 52(2), 119-137 (1990)

27. Cădariu, L, Radu, V: Stability properties for monomial functional equations. An. Univ. Vest. Timiş., Ser. Mat.-Inform. 43(1), 23-38 (2005)

28. Cădariu, L, Radu, V: Remarks on the stability of monomial functional equations. Fixed Point Theory 8(2), 201-218 (2007)

29. Eungrasamee, T, Udomkavanich, $P$, Nakmahachalasint, $P$ : Generalized stability of classical polynomial functional equation of order $n$. Adv. Differ. Equ. (2012). doi:10.1186/1687-1847-2012-135

30. Gilányi, A: Hyers-Ulam stability of monomial functional equations on a general domain. Proc. Natl. Acad. Sci. USA 19 10588-10590 (1999)

31. Gilányi, A: On the stability of monomial functional equations. Publ. Math. (Debr.) 58(1-2), 201-212 (2000)

32. Lee, YH: On the stability of the monomial functional equation. Bull. Korean Math. Soc. 45(2), 397-403 (2008)

33. Mirmostafaee, A: Stability of the monomial functional equation in quasi normed spaces. Bull. Korean Math. Soc. 47(4), 777-785 (2010)

34. Baker, J: A general functional equation and its stability. Proc. Am. Math. Soc. 133, 1857-1864 (2005)

35. Cholewa, P: Almost approximately polynomial functions. In: Nonlinear Analysis, pp. 127-136. World Scientific Singapore (1987)

36. Gajda, Z: A solution to a problem of J. Schwaiger. Aequ. Math. 32(1), 38-44 (1987)

37. Ger, R: On almost polynomial functions. Colloq. Math. 24, 95-101 (1971)

38. Kominek, Z: A few remarks on almost C-polynomial functions. Math. Slovaca 55(5), 555-561 (2005)

39. Popa, D, Raşa, I: The Fréchet functional equation with application to the stability of certain operators. J. Approx. Theory 184(1), 138-144 (2012)

40. Schwaiger, J: Functional equations for homogeneous polynomials arising from multilinear mapings and their stability. Ann. Math. Sil. 8, 157-171 (1994)

41. Székelyhidi, L: Note on a stability theorem. Can. Math. Bull. 25(4), 500-501 (1982)

42. Székelyhidi, L: Fréchet's equation and Hyers theorem on noncommutative semigroups. Ann. Pol. Math. 48, 183-189 (1988)

43. Tabor, J: k-proper families and almost approximately polynomial functions. Glas. Mat. 38(58), 177-191 (2001)

44. Wolna, D: The stability of monomial functions on a restricted domain. Aequ. Math. 72(1-2), 100-109 (2006)

10.1186/1687-1847-2014-16

Cite this article as: Dăianu: Recursive procedure in the stability of Fréchet polynomials. Advances in Difference Equations 2014, 2014:16

\section{Submit your manuscript to a SpringerOpen ${ }^{\ominus}$ journal and benefit from:}

- Convenient online submission

Rigorous peer review

- Immediate publication on acceptance

- Open access: articles freely available online

- High visibility within the field

- Retaining the copyright to your article 